Applied normal force

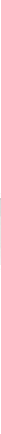




\title{
Tribochemical synthesis of nano-lubricant films from adsorbed molecules at sliding solid interface: Tribo-polymers from $\alpha$-pinene, pinane, and n-decane
}

\author{
Xin He, Anthony J. Barthel, and Seong H. Kim* \\ Department of Chemical Engineering, Materials Research Institute, \\ Pennsylvania State University, University Park, PA 16802, USA \\ * Corresponding author: shkim@engr.psu.edu
}

\begin{abstract}
:
The mechanochemical reactions of adsorbed molecules at sliding interfaces were studied for $\alpha$ pinene $\left(\mathrm{C}_{10} \mathrm{H}_{16}\right)$, pinane $\left(\mathrm{C}_{10} \mathrm{H}_{18}\right)$, and n-decane $\left(\mathrm{C}_{10} \mathrm{H}_{22}\right)$ on a stainless steel substrate surface. During vapor phase lubrication, molecules adsorbed at the sliding interface could be activated by mechanical shear. Under the equilibrium adsorption condition of these molecules, the friction coefficient of sliding steel surfaces was about 0.2 and a polymeric film was tribochemically produced. The synthesis yield of $\alpha$-pinene tribo-polymers was about twice as much as pinane tribo-polymers. In contrast to these strained bicyclic hydrocarbons, n-decane showed much weaker activity for tribo-polymerization at the same mechanical shear condition. These results suggested that the mechanical shear at tribological interfaces could induce the opening of the strained ring structure of $\alpha$-pinene and pinane, which leads to polymerization of adsorbed molecules at the sliding track. On a stainless steel surface, such polymerization reactions of adsorbed molecules do not occur under typical surface reaction conditions. The mechanical properties and boundary lubrication efficiency of the produced tribo-polymer films are discussed.
\end{abstract}

Key words: Mechanochemical reaction; Tribo-polymer; Lubrication; Mechanical properties 


\section{Introduction}

Lubrication is necessary for mechanical devices to prevent wear of sliding parts and increase the device operation reliability. Mechanical systems with sliding contacts often fail due to surface wear at the sliding interface.[1] To avoid this problem, protective coatings have been applied, yet the durability of surface coatings often limits the reliability of such approaches employing one-time application of lubricant coatings.[2,3] As an alternative, lubrication by molecular adsorbate films in equilibrium with the vapor phase has been developed; this method is called vapor phase lubrication (VPL).[4-6]

In VPL, the vapor molecules from the surrounding environment adsorb on the surface to form mono- or multilayers, which can prevent wear.[7] Just like coatings, the adsorbed molecules might be removed (desorbed) during sliding, yet as long as the vapor pressure of the adsorbate is kept above a certain level, the lubricant molecules could adsorb to its equilibrium thickness to replenish the film; thus, lubrication with self-healing can be possible. Alcohol has been demonstrated to be an effective lubricant for various kinds of materials.[7-9] VPL has also been demonstrated to be effective for lubrication of microelectromechanical systems (MEMS) without interfering with the device function or performance.[10]

One limitation of VPL is the fact that the vapor pressure of lubricating molecules must be kept above a certain level at all times so that the minimum thickness of adsorbate films is assured. The minimum thickness is typically a monolayer.[11] In the case of n-pentanol, when its vapor pressure is reduced to less than $10 \%$ of the saturation vapor pressure, the surface could wear due to insufficient supply of lubricant molecules.[4] When this happens on a silicon substrate, the wear exposes dangling bonds of silicon at the damaged surface, which could induce chemical reactions of n-pentanol molecules, producing tribo-polymers at the wear track.[12] These tribo- 
polymers formed from n-pentanol could provide lubrication effects in the absence of n-pentanol vapor, but its lubrication effect diminished too quickly. Also, the formation of tribo-polymers from n-pentanol requires reactive surface sites which may not be available, especially on welllubricated interfaces.

During VPL, molecules adsorbed at the sliding solid-solid interface might undergo mechanochemical or tribochemical reactions under high contact pressure and frictional shear force.[13,14] In these cases, chemical reaction is activated by mechanical energy, rather than thermal or photochemical origins.[15] Under mechanical compression or shear, the potential energy surface of molecules may be distorted and the energy barrier be lowered along a reaction coordinate, $[16,17]$ expediting or allowing chemical reactions that would not occur under thermal or photochemical conditions.[13,14,18] Recently, it has been shown that allyl alcohol adsorbed on stainless steel could be polymerized under interfacial shear conditions.[19] Even though allyl alcohol has a double bond, it does not undergo typical radical polymerization in thermal reaction conditions.[20] However, it was easily polymerized under the mechanical shear condition. More interestingly, the tribo-polymer produced from allyl alcohol at the sliding track acted as a boundary lubrication film for an extended period of time without continuous supply of lubricant vapor.[19]

In this paper, we report tribochemical reactions of $\alpha$-pinene $\left(\mathrm{C}_{10} \mathrm{H}_{16}\right)$ and pinane $\left(\mathrm{C}_{10} \mathrm{H}_{18}\right)$. These are bicyclic terpenes with highly-strained 4-membered rings. $\alpha$-pinene is the major constituent of pine tree oil or turpentine and is known to undergo the ring opening of the strained 4-membered ring to generate a relatively stable tertiary pinane carbocationic species and produce polymers.[21,22] The viscoelastic properties of poly- $\alpha$-pinene is suitable for a tackifying agent of pressure-sensitive adhesives.[23] The same property could be used for boundary lubrication of 
sliding interfaces. It was hypothesized that the mechanical shear at tribological interfaces could assist the ring opening or rearrangement leading to the production of tribo-polymeric films at the sliding track during VPL. The mechanochemical or tribochemical reaction activity of these strained bicyclic molecules was compared with n-decane $\left(\mathrm{C}_{10} \mathrm{H}_{22}\right)$, a linear alkane molecule without any internal strain. The viscoelastic property and boundary lubrication efficiency of the produced tribo-polymer films were studied.

\section{Experimental Details}

A custom-made reciprocating ball-on-flat tribometer with an environment control capability was employed to test friction and wear between an AISI 440C stainless steel substrate and an AISI 440C stainless steel ball (diameter $=3 \mathrm{~mm}$ ). The substrate was polished with fine grit sandpapers and a polishing solution with $1 \mu \mathrm{m}$ colloidal alumina. Bearing-grade 440C balls with smooth surface finish were purchased from McMaster-Carr (Ohio, USA). All substrate and ball surfaces were cleaned with ethanol followed by UV/ozone. The friction and wear tests were performed with a contact load of $2 \mathrm{~N}$, which corresponded to a Hertzian contact pressure of about $0.6 \mathrm{GPa}$. The sliding speed was $0.4 \mathrm{~cm} / \mathrm{s}$ and the sliding span was $2.5 \mathrm{~mm}$. After the friction test, the slide track was analyzed in ambient air by optical profilometry (Zygo NewView 7300).

The vapor control during the friction and wear test was done in a continuous flow mode. Details of the vapor control system were previously described.[24] The liquids used to create vapor environments were (+)- $\alpha$-pinene (2,6,6-trimethylbicyclo[3.1.1]hept-2-ene; CAS No. 7785-

70-8), pinane (2,6,6-trimethylbicyclo[3.1.1]heptane; CAS No. 473-55-2), and n-decane (CAS No. 124-18-5). The saturated organic vapor flow was created by bubbling $\mathrm{N}_{2}$ carrier gas through a 
flask filled with the organic liquid and glass beads. The saturation vapor pressure $\left(p_{\text {sat }}\right)$ of $\alpha-$ pinene, pinane, and n-decane is 4.75 Torr, 2.19 Torr, and 1.43 Torr, respectively, at room temperature. The saturated vapor stream was mixed with the dry $\mathrm{N}_{2}$ carrier gas to obtain the desired partial pressure of the vapor. The partial pressures of the organic vapors are expressed as percent relative to saturation $\left(p / p_{\text {sat }} \times 100 \%\right)$. In this experiment, we applied $30 \% \mathrm{p} / p_{\text {sat }}$ vapor pressure of $\alpha$-pinene, pinane, and $\mathrm{n}$-decane, which appears to be high enough to form a coverage close to the monolayer on stainless steel surface.[7]

The adsorption of organic vapor was monitored with polarization-modulation reflection absorption infrared spectroscopy (PM-RAIRS). A ThermoNicolet Nexus 670 spectrometer with a Hinds Instrument photoelastic modulator was used for PM-RAIRS experiments. PM-RAIRS probes the adsorption layer through absorption of IR beam sequentially modulated with s- and ppolarizations.[25] The metal selection rule of IR reflection allows detection of the adsorbed layer without the interference of the gas phase.[26] The sum $(\mathrm{p}+\mathrm{s})$ signal of these two polarizations carries the signal of both adsorbate and gas phase molecules while the difference $(p-s)$ signal contains only the vibration spectrum of the adsorbate molecules. Consequently, the ratio $\left(p^{-s}\right) /(p+s)$ represents only the vibration spectrum of adsorbate layer. In this experiment, the PM-RAIRS measurement was conducted on copper surface, instead of stainless steel surface, because copper gives stronger reflection signals.[27,28] The PM-RAIRS data of near-saturation vapor pressure is shown instead of the $30 \% \mathrm{p} / \mathrm{p}_{\text {sat }}$ spectra for better clarity with a higher signal to noise ratio; the spectral features in those spectra were the same except the total signal intensity. The full adsorption isotherm behaviors of the molecules used in this experiment are shown in the Supplementary Information.[7,29-31] 
The tribo-polymers formed and accumulated at both ends of the slide tracks were analyzed with micro-IR spectroscopy and time-of-flight secondary ion mass spectrometry (ToFSIMS). Micro-IR analysis was carried out using a Bruker Hyperion 3000 IR Microscope coupled to IFS 66/s spectrometer with a $15 \mathrm{X}$ objective lens. The analyzed areas were $50 \mu \mathrm{m}^{2}$. ToF-SIMS analysis was conducted using a Physical Electronics nanoToF II equipped with a bismuth cluster liquid metal ion gun, a triple focusing electrostatic analyzer, and a multichannel plate (MCP) detector. $\mathrm{A} \mathrm{Bi}_{3}{ }^{2+}$ ion beam $\left(30 \mathrm{keV}\right.$; total dose $=6.95 \times 10^{12}$ ions $\left./ \mathrm{cm}^{2}\right)$ was used to sputter a $70 \times$ $70 \mu \mathrm{m}^{2}$ area of interest. The mass-to-charge ratios of the ejected secondary ions were measured to investigate the molecular composition of tribo-polymers.

The tribo-polymers around the slide track were imaged with atomic force microscopy (AFM) using a Digital Instrument MultiMode scanning probe microscope. Silicon AFM tips (NANOSENSORSTM PPP-FM) were cleaned with UV/ozone to remove any residual contaminant species. The spring constants of the AFM cantilevers were calibrated using the Sader method; they were $2.5 \mathrm{~N} / \mathrm{m}$ for the AFM cantilever used for $\alpha$-pinene test, $2.8 \mathrm{~N} / \mathrm{m}$ for pinane test, and $2.7 \mathrm{~N} / \mathrm{m}$ for n-decane test.[32] Topographic images of tribo-products were obtained with a tapping-mode scanning. The scan size of the AFM images was $90 \mu \mathrm{m} \times 90 \mu \mathrm{m}$. The viscoelastic properties of the tribo-polymer were investigated by recording the normal deflection signal of the cantilever during the tip approach and retraction with different set values of final indentation force $(20-120 \mathrm{nN})$. The load and unload rate of the vertical piezo-scanner during the indentation test was $440 \mathrm{~nm} / \mathrm{s}$. The tip-surface distance or indentation depth of the sample was calculated by subtracting the cantilever defection distance from the total travel distance of the piezo-scanner.[33] 


\section{Result and Discussion}

Friction and wear were measured between the AISI 440C stainless steel ball and substrate under $30 \% \mathrm{p} / p_{\text {sat }} \alpha$-pinene, pinane, and $\mathrm{n}$-decane vapors. As shown in Figure 1a, the unlubricated friction was very high and noisy in dry nitrogen. Because there was no lubricant between sliding interfaces, both ball and substrate showed severe wear (Figure 1b). In contrast, tribo-tests in $30 \% \mathrm{p} / p_{\text {sat }}$ of $\alpha$-pinene, pinane, and n-decane vapors showed a friction coefficient of $\sim 0.2$ that remained stable for the duration of test. This friction coefficient value is in good agreement with the typical values observed for VPL with organic molecules.[7,10] After 600 reciprocating cycles of friction tests, deposits with iridescent color could be seen at the ends of the sliding tracks in optical microscope images (Figure 1a). The iridescent color was produced due to the interference of the light reflected from the top and bottom surfaces of the deposit on the substrate surface. The surface of the deposit looked smooth, suggesting that they are not wear debris or particulates of substrate materials. These features in the optical images indicated that the deposits must be polymeric species with varying thickness and smooth surfaces formed during the friction test in $\alpha$-pinene, pinane and n-decane vapors.

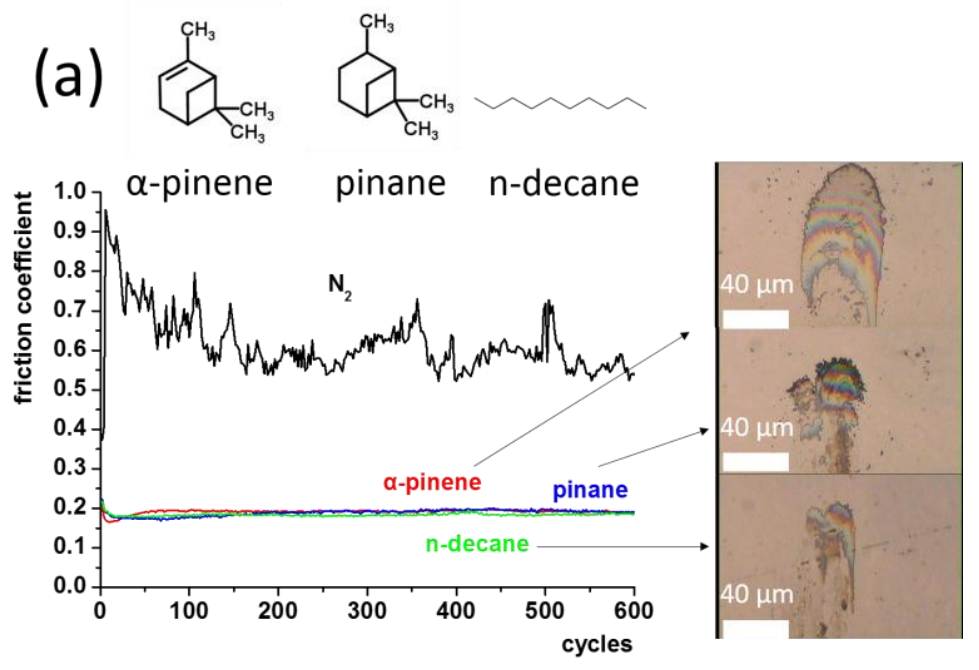



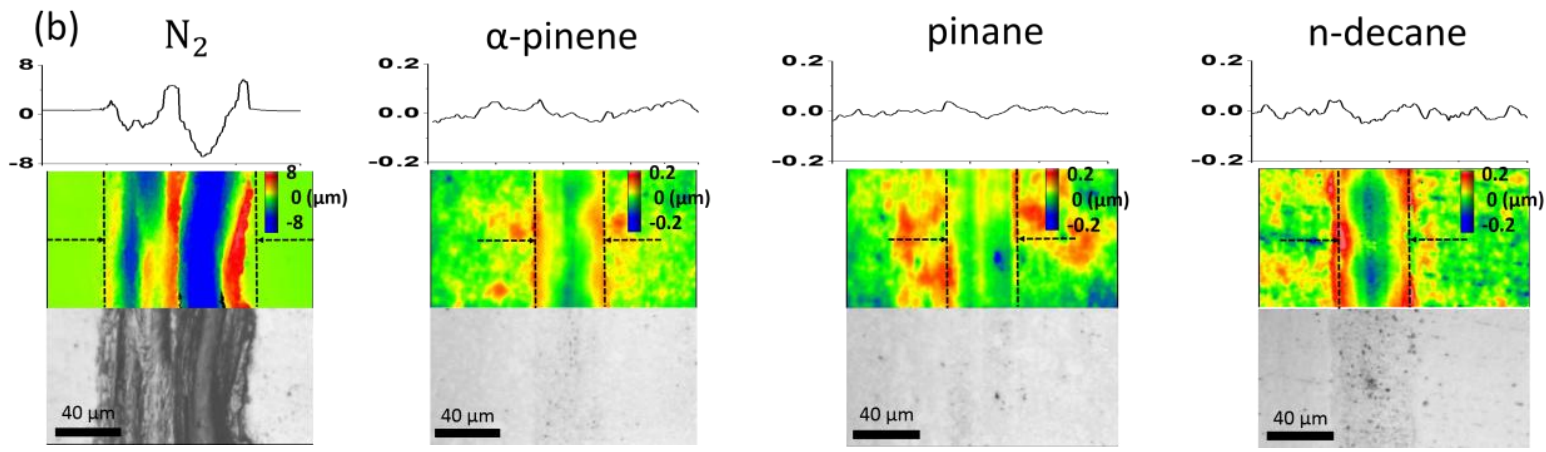

Figure 1. (a) Friction coefficient of stainless steel in dry $\mathrm{N}_{2}$ and VPL with $30 \% \mathrm{p} / p_{\text {sat }} \alpha$-pinene, pinane, and n-decane; (b) Cross-section line profile (top), optical profilometry image (middle) and optical microscope image (bottom) of wear tracks after friction tests in dry $\mathrm{N}_{2}$ as well as VPL with $\alpha$-pinene, pinane, and n-decane. In (b), tribo-polymers were removed by ethanol to analyze the damage in the substrate surface. The unit of the y-axis of the cross-section line profile is $\mu \mathrm{m}$.

The lubrication and wear prevention effects of the adsorbed vapors under equilibrium adsorption conditions were confirmed by analyzing the wear track with optical profilometry. Figure $1 \mathrm{~b}$ presents the line profiles as well as three-dimensional images of the wear tracks. In dry $\mathrm{N}_{2}$ environment, the wear marks on the substrate were $\sim 8 \mu \mathrm{m}$ deep and $\sim 80 \mu \mathrm{m}$ wide. In contrast, there were no discernible wear mark in the slide tracks tested in $\alpha$-pinene and pinane vapors when polymeric deposits were removed by rinsing and rubbing with a cotton swap wet with ethanol. A slight depression ( $<10 \mathrm{~nm}$ deep, $\sim 40 \mu \mathrm{m}$ wide) could be identified in the line profile (Figure 1b). The inner surface of this depressed track was very smooth. This depression was caused by the plastic deformation of the substrate due to the applied load, not due to mechanical wear of the slide track. In the case of n-decane, the accumulation of polymeric deposits was much smaller compared to the $\alpha$-pinene and pinane VPL cases. Although the wear track tested in 
the n-decane VPL had dark spots in optical microscope images, the topographic analysis indicated that the substrate wear was also negligible (Figure 1b).
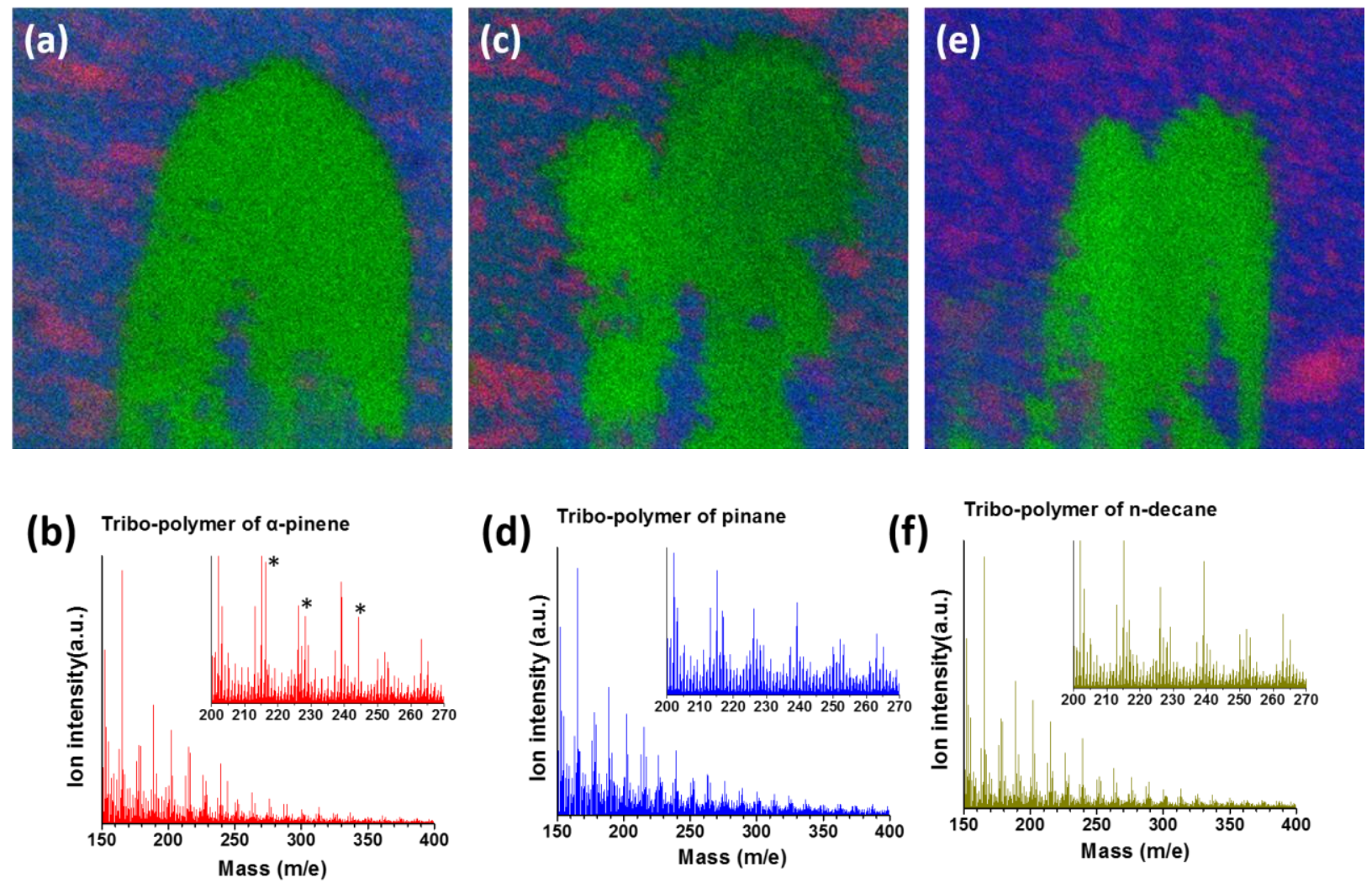

Figure 2. ToF-SIMS ion maps (a,c,e) and mass spectra (b,d,f) and of tribo-polymer products deposited at the end of the slide track produced during (a,b) $\alpha$-pinene VPL, (c,d) pinane $\alpha$ pinene, (e,f) n-decane VPL. In the ion maps, blue is the iron ion $(\mathrm{m} / \mathrm{e}=55.93 \mathrm{amu})$, red is the chromium ion $(\mathrm{m} / \mathrm{e}=51.94 \mathrm{amu})$, and green is the total intensity of ions with $\mathrm{m} / \mathrm{e}=164 \sim 300 \mathrm{amu}$. The mass of ions marked with * in the inset of (b) are 216, 228, and $244 \mathrm{amu}$.

The polymeric nature of the reaction product was confirmed with ToF-SIMS. Figure 2 shows the selected ion maps of the reaction products piled at the ends of the sliding tracks as well as the mass spectra of ions desorbed upon sputtering with $\mathrm{Bi}_{3}{ }^{2+}$ ions. The images of the 
ToF-SIMS ion maps were very similar to the optical images shown in Figure 1a. Outside the polymeric deposit region, the main ions detected were iron $(\mathrm{m} / \mathrm{e}=55.93 \mathrm{amu})$ and chromium $(\mathrm{m} / \mathrm{e}=51.94 \mathrm{amu})$ which are the main constituents of the stainless steel surface. Within the deposit region, these inorganic ions from the substrate were not detected. Instead, organic ion peaks were detected with masses extending up to $\sim 500 \mathrm{amu}$. Note that the molecular weights of the adsorbed $\alpha$-pinene, pinane, and n-decane molecules are $136 \mathrm{amu}, 138 \mathrm{amu}$, and $144 \mathrm{amu}$, respectively. Also, the ions with masses higher than the molecular weight of the adsorbed molecules were separated by $13 \mathrm{amu}(\mathrm{CH})$ and $14 \mathrm{amu}\left(\mathrm{CH}_{2}\right)$, consistent with fragmentation of polymeric hydrocarbons.[4], [10] These ToF-SIMS data clearly indicated the presence of organic molecules whose molecular weights are higher than $500 \mathrm{amu}$. Due to heavy fragmentation of organic species by high energy ion sputtering and low detection yield of the MCP detector for higher mass organic ions, the maximum molecular weights of the polymeric species could not be determined. It was reported that typical molecular weight of poly- $\alpha$-pinene produced by cationic polymerization is 700-800 amu.[21] There are no references for molecular weight comparison of the polymers produced from pinane and n-decane since these molecules cannot be polymerized via chemical initiators. The ToF-SIMS results confirmed that the reaction products detected around the sliding track are polymers. Since the stainless steel surface cannot induce such polymerization reactions of the adsorbed molecules at ambient conditions, they must be produced via tribochemical or mechanochemical mechanisms. Thus, they will be called tribopolymers henceforth.

In order to determine the exact molecular structure of tribo-polymers, nuclear magnetic resonance (NMR) analysis is required. But the amount of tribo-products synthesized during the friction test was too small for NMR analysis.[21,34] This is one of the main challenges in 
studying tribochemical reactions. Although not as definitive as NMR, IR spectroscopy can provide qualitative information of the molecular structure of tribo-products. In the previous adsorption isotherm study of n-decane using PM-RAIRS, it was found that a complete monolayer may not form on a flat surface at ambient conditions with partial vapor pressure lower the saturation condition.[7] However, capillary condensation around the periphery of the contact may allow formation of at least a monolayer of adsorbed molecules to lubricate the contacts.[29,35,36] The organic reaction product can also be detected with using micro-IR spectroscopy.[19,37]
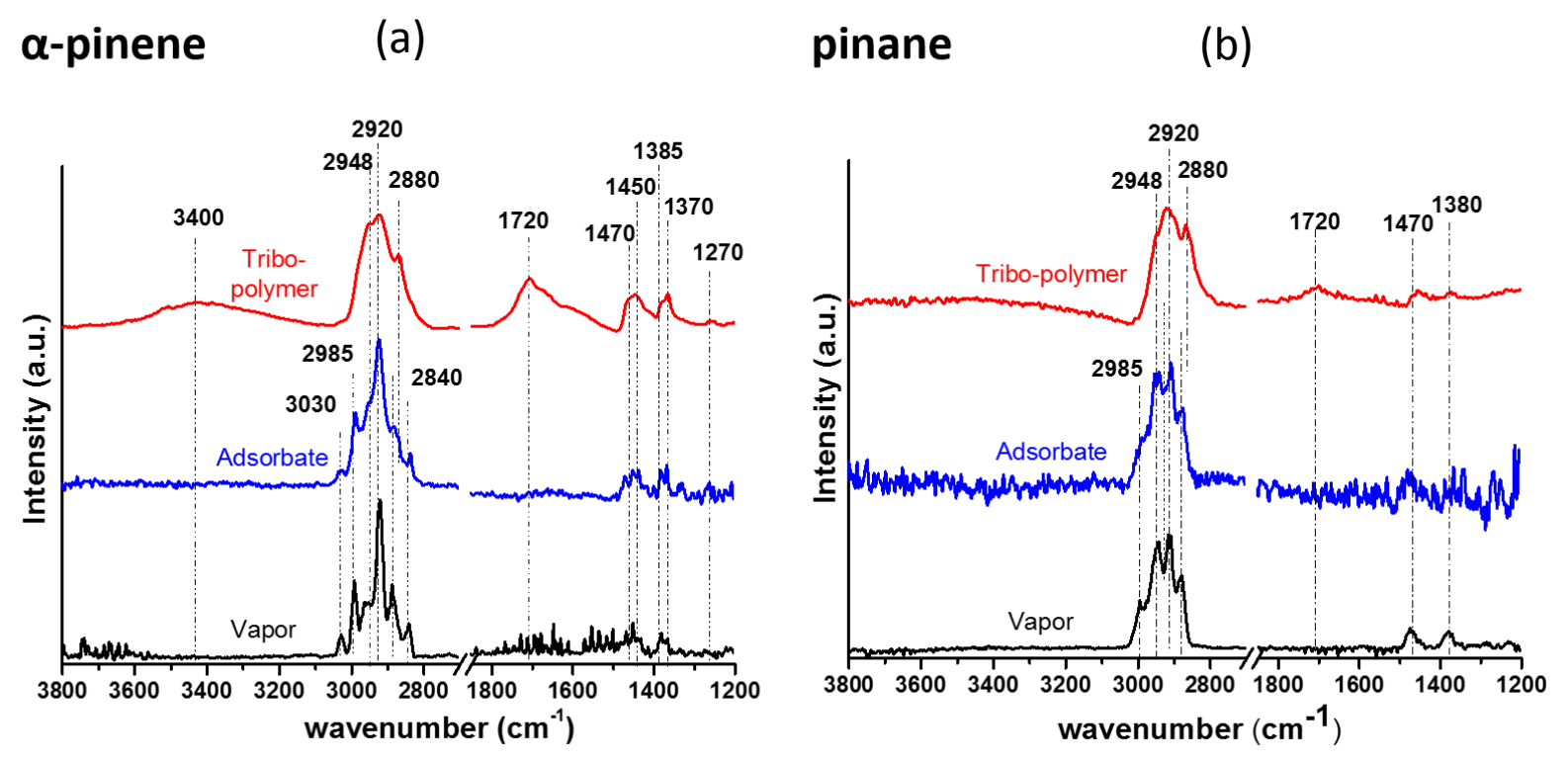

Figure 3. Infrared spectra of vapor, adsorbate, and tribo-polymer of (a) $\alpha$-pinene and (b) pinane.

The vapor spectra were the sum signal of PM-RAIRS measurements; the adsorbate spectra were the difference signal of PM-RAIRS normalized with the sum signal. The tribo-polymer spectra were obtained from the deposits within a $50 \times 50 \mu \mathrm{m}^{2}$ area at the end of the sliding track. 
Figure 3 compares the IR spectra of $\alpha$-pinene and pinane adsorbates and their tribopolymers with the vapor phase spectra of these molecules. In Figure 3a, the IR spectrum of $\alpha$ pinene vapor shows peaks at $1270 \mathrm{~cm}^{-1}, 1370 \mathrm{~cm}^{-1}, 1385 \mathrm{~cm}^{-1}, 1450 \mathrm{~cm}^{-1}$, and $1470 \mathrm{~cm}^{-1}$ in the $\mathrm{C}-\mathrm{H}$ bending vibration region and $2840 \mathrm{~cm}^{-1}, 2880 \mathrm{~cm}^{-1}, 2910 \mathrm{~cm}^{-1}, 2945 \mathrm{~cm}^{-1}, 2985 \mathrm{~cm}^{-1}$, and $3030 \mathrm{~cm}^{-1}$ in the $\mathrm{C}-\mathrm{H}$ stretch region. [38] The small peak at $3030 \mathrm{~cm}^{-1}$ is characteristic to the $=\mathrm{C}$ $\mathrm{H}$ vibrations of $\alpha$-pinene. The $\mathrm{C}=\mathrm{C}$ stretch band at $1660 \mathrm{~cm}^{-1}$ were difficult to identify due to its weak intensity and the interference from gas-phase water signals (sharp spikes in $1400-1800$ $\mathrm{cm}^{-1}$ ). The $2985 \mathrm{~cm}^{-1}$ peak is the asymmetric stretch of the $\mathrm{CH}_{3}$ group at the strained 4membered ring. This peak position is higher than typical values of the $\mathrm{CH}_{3}$ asymmetric stretch modes; it is well known that the stretch vibration position of the $\mathrm{CH}_{3}$ groups attached to the strained ring is blue-shifted.[39,40] The adsorbate spectrum of $\alpha$-pinene showed the peaks identical to the vapor phase spectrum, indicating that the adsorbed $\alpha$-pinene on the stainless steel surface remains intact without any dissociation through chemical reactions with the stainless steel surface.

The $\alpha$-pinene tribo-polymer exhibited the $\mathrm{C}-\mathrm{H}$ bending peaks almost identical to the molecular $\alpha$-pinene, but the $\mathrm{C}$-H stretch peaks were changed drastically. Especially, the 2985 $\mathrm{cm}^{-1}$ and $3030 \mathrm{~cm}^{-1}$ peaks disappeared, indicating that the strained 4-membered ring to which two $\mathrm{CH}_{3}$ groups are attached and the $\mathrm{C}=\mathrm{C}$ double bond in the heptene ring are converted during the tribo-polymerization reactions. The relative intensities of $2880 \mathrm{~cm}^{-1}, 2920 \mathrm{~cm}^{-1}$, and $2948 \mathrm{~cm}^{-}$ ${ }^{1}$ peaks were very similar to poly- $\alpha$-pinene produced via cationic polymerization.[41] The broad peaks at $3400 \mathrm{~cm}^{-1}$ and $1720 \mathrm{~cm}^{-1}$ were hydroxyl and carbonyl peaks. These groups were not present in the adsorbate spectrum. It is known that the unsaturated double bond of poly- $\alpha$-pinene can be easily oxidized in air, producing hydroxyl and carbonyl groups.[41] The hydroxyl and 
carbonyl groups in the $\alpha$-pinene tribo-polymer is believed to be formed during the air exposure of the sample inevitably happened during the sample transfer from the tribo-test cell to the IR spectrometer; thus, the presence of hydroxyl and carbonyl groups supported that the $\alpha$-pinene tribo-polymer has the molecular functional groups or structures similar to the chemicallysynthesized poly- $\alpha$-pinene. In ToF-SIMS analyses (Figure 2), it was noted that ions with $\mathrm{m} / \mathrm{e}=$ $216 \mathrm{amu}, 228 \mathrm{amu}$, and $244 \mathrm{amu}$ were prominent in the mass spectrum of the $\alpha$-pinene tribopolymer (marked with * in the inset of Figure $2 b$ ), compared to those of the pinane and n-decane tribo-products. Since their mass differences are $12 \mathrm{amu}$ and $16 \mathrm{amu}$, they must correspond to fragment ions containing carbonyl $(\mathrm{C}=\mathrm{O})$ groups, consistent with the presence of the $1720 \mathrm{~cm}^{-1}$ peak in IR (Figure 3a). These results indicated that although initiation and propagation mechanisms are different, the tribochemical reactions of $\alpha$-pinene produce polymers having oxidation behaviors similar to the poly- $\alpha$-pinene synthesized through cationic polymerization.

In Figure 3b, the IR spectrum of pinane vapor has the peaks at $2880 \mathrm{~cm}^{-1}, 2910 \mathrm{~cm}^{-1}$, and $2940 \mathrm{~cm}^{-1}$ as well as a shoulder peak at $2985 \mathrm{~cm}^{-1}$ in the C-H stretch region and two peaks at $1380 \mathrm{~cm}^{-1}$ and $1470 \mathrm{~cm}^{-1}$ in the $\mathrm{C}-\mathrm{H}$ bending region. Pinane has the strained 4-membered ring but does not have the $\mathrm{C}=\mathrm{C}$ bond; thus, the presence of the $2985 \mathrm{~cm}^{-1}$ peak but the absence of the $3030 \mathrm{~cm}^{-1}$ are consistent with the molecular structure of pinane. Again, the IR spectrum of the adsorbed pinane has the same peaks of the pinane vapor, indicating that the adsorbed species are molecularly intact. Since pinane is not readily polymerized chemically, no reference spectrum could be found in the literature. However, it was noted that all peaks of the pinane tribo-polymer were the same as those observed for the $\alpha$-pinene tribo-polymer, except that the peak intensities at $3400 \mathrm{~cm}^{-1}$ and $1720 \mathrm{~cm}^{-1}$ were much lower. The absence of the $2985 \mathrm{~cm}^{-1}$ peak in the pinane tribo-polymer spectrum indicated that the strained 4-membered ring of pinane must be 
dissociated and involved in polymerization reactions. These results suggested that the pinane tribo-polymer must have molecular structure similar to the $\alpha$-pinene tribo-polymer, except the $\mathrm{C}=\mathrm{C}$ double bonds which are subject to oxidations in air.

The exact initiation mechanism for tribochemical polymerization (Figure 2) could not be determined unambiguously; but it must originate from mechanochemical processes since no chemical initiator was used and the vapor molecules were physisorbed without dissociation on the stainless steel surface (Figure 3). There was no wear, other than small plastic deformation, of the stainless steel substrate (Figure 1). Thus the exposure of reactive dangling bonds by substrate wear, which was the key requirement for tribo-polymer formation from n-pentanol adsorbed on a silicon wafer,[37] is not responsible for the tribo-polymerization of vapor molecules studied in this work. Since the sliding speed was very low in our test condition, frictional heating of the slide track was negligible. Based on the thermal diffusivity of stainless steel and the measured friction coefficient $(\sim 0.2)$, the temperature rise of the sliding contact was estimated to be less than one degree (see Supplementary Information).[42] Ruling out these possibilities, one could attribute the activation and initiation of the adsorbed molecule for polymerization to a mechanochemical or tribochemical process.

Under mechanical stress or shear, the potential energy of chemical bonds could be distorted, facilitating chemical reactions that would not occur under normal conditions. $[15,16,43,44]$ Based on the IR analysis (Figure 3), it could be proposed that the C-H or $\mathrm{C}-\mathrm{C}$ bonds of the highly-strained 4-membered rings of $\alpha$-pinene and pinane are the sites most likely to be activated upon shear of molecules at the sliding solid interface. Note that even though n-decane does not have internal strain, it was also polymerized tribochemically at the sliding interface (Figure 2f). The mechanical energy at the shearing interface must be high 
enough to dissociate $\mathrm{C}-\mathrm{C}$ and or $\mathrm{C}-\mathrm{H}$ bonds. The presence of ring strain or $\mathrm{C}=\mathrm{C}$ double bond could facilitate these dissociation processes. This would release the strain of the 4-membered rings of these molecules, forming relatively stable tertiary ions or radicals.[21,22,45]

Scheme 1 illustrates the intermediate species involved in the cationic polymerization of $\alpha$-pinene. In the case of $\alpha$-pinene, the proton donation from the cationic initiator produces tertiary carbenium ion with the pinane structure.[21,22,45] Then, the pinane type cation isomerizes to the $p$-menthane and bornane type ions, which undergo propagation reactions forming polymers. The mechanochemical ring-opening of $\alpha$-pinene would produce ions or radicals whose structures are similar to the $p$-menthane and bornane type cations shown in Scheme 1. Since these intermediates are confined and mixed with monomers within the physical contact region between two solid surfaces, they could undergo polymerization reactions. In the case of pinane, the main intermediate would be the bornane type species; thus, the produced tribo-polymer would not contain double bonds, which makes it less susceptible to oxidation.

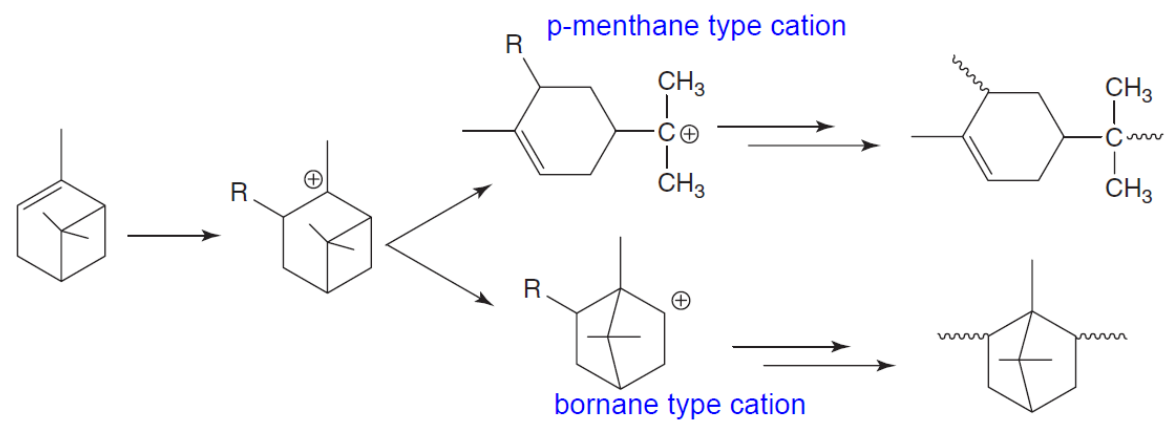

Scheme 1. Polymerization of $\alpha$-pinene via cationic intermediates. [45]

The tribo-polymerization yield was estimated by measuring the volume of the tribopolymer deposits produced during 600 reciprocating cycles under VPL conditions with AFM. 
Figure 4 displays the $90 \mu \mathrm{m} \times 90 \mu \mathrm{m}$ AFM images of tribo-polymers piled at both ends and the middle of the slide tracks after 600 cycles of VPL tests. From the line profiles shown in the left of the AFM images, it can be seen that the tribo-polymer products were mostly pushed and piled at the turning points of the ball sliding action. In the middle of wear track, the tribo-polymers were squeezed to the side of the track. By integrating the line profiles over the entire sliding track, the total amounts of the tribo-polymer product produced during the 600 sliding cycles were estimated to be approximately $7.0 \times 10^{3} \mu \mathrm{m}^{3}, 3.2 \times 10^{3} \mu \mathrm{m}^{3}$, and $0.5 \times 10^{3} \mu \mathrm{m}^{3}$ for $\alpha$-pinene, pinane, and n-decane VPL, respectively. The large difference in polymerization yields between strained bicyclic molecules ( $\alpha$-pinene and pinane) versus linear alkane molecule (n-decane) supported the hypothesis that the strained ring can easily be activated mechanochemically. The higher polymerization yield for $\alpha$-pinene than pinane must be due to the activation of the $\mathrm{C}=\mathrm{C}$ double bond or additional ring strain due to the presence of the $\mathrm{C}=\mathrm{C}$ double bond in the heptene ring. 


\section{(a)}

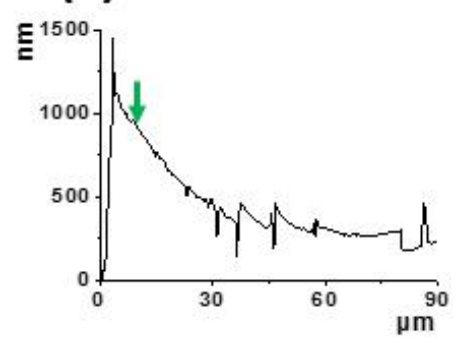

(b)

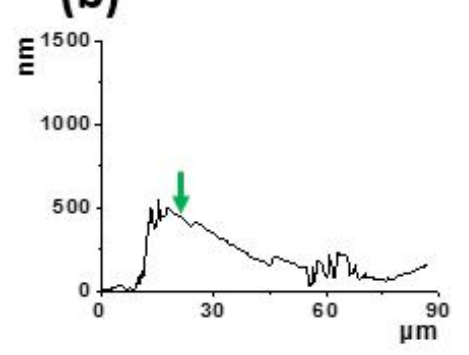

(c)

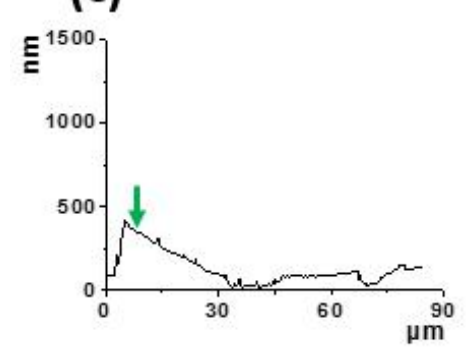

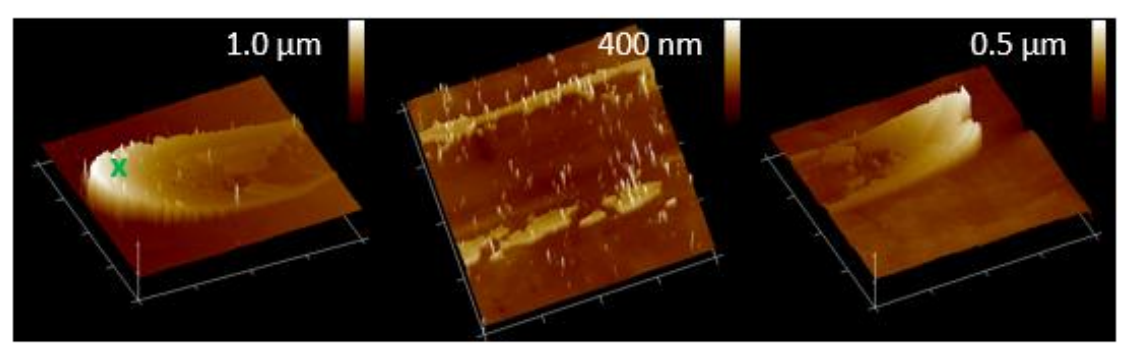
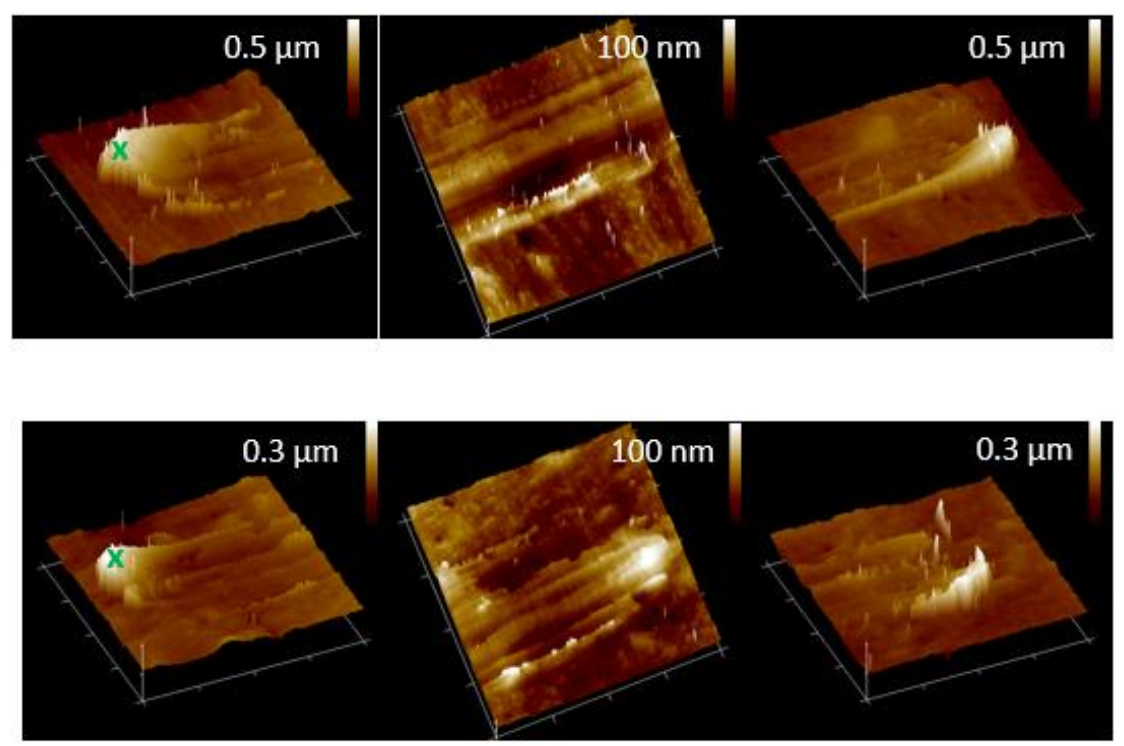

Figure 4. Tapping-mode AFM images and line profiles of tribo-polymers piled at the ends and sides of the slide track after VPL tests in (a) $\alpha$-pinene, (b) pinane, and (c) n-decane vapors. The line profile was taken along the sliding direction at the left end of the slide track. Each AFM image was taken over a $90 \mu \mathrm{m} \times 90 \mu \mathrm{m}$ area. Indentation test positions were marked as green cross mark on the AFM images and arrow on the cross-section profiles.

The mechanical properties of tribo-polymers produced from $\alpha$-pinene, pinane, and ndecane were investigated with AFM by recording the force as a function of tip-surface distance during the indentation load and unload cycle (Figure 5).[33,46-48] The indentation positions were chosen to be on the tribo-polymer piled up at the end of the wear track, which are marked 
in Figure 4. In that way, the indentation depth did not exceed a few percent of the tribo-polymer thickness to avoid any contribution from the solid surface.[49] The zero indentation position was set as the cross-points of the extrapolated lines of the linear portions of the loading and freestanding curves. It is noted that the force curve started increasing even before the tip surface distance became zero. This was due to the polymer films transferred to the tip surface during the tapping mode imaging (see Supplementary Information). In this plot, the slope of the load and unload curve corresponds to the stiffness of the material being probed with the AFM tip and the largest negative force measured is the adhesion force between the tip and the probed surface.

(a)

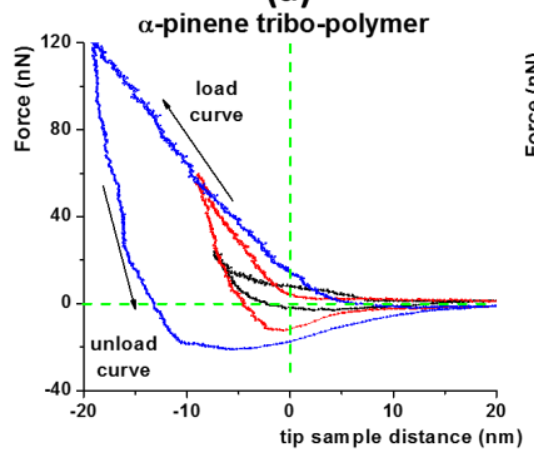

(b)

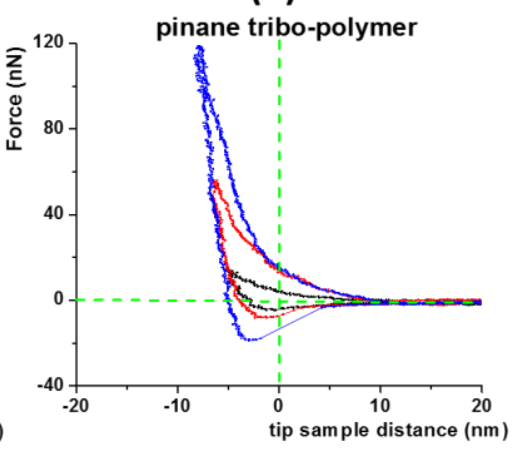

(c)

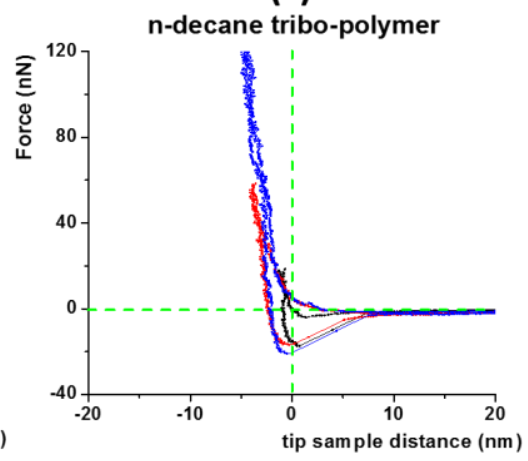

Figure 5. Force-distance curve of (a) $\alpha$-pinene tribo-polymers, (b) pinane tribo-polymers and (c) n-decane tribo-polymers accumulated at the end of slide track on stainless steel. Each tribopolymer was probed with a new clean AFM tip. The piezo-scanner travel distance was converted to the tip-sample distance by subtracting the cantilever deflection distance. The tips was used for tapping-mode imaging before the force-distance measurements.

The stiffness of the $\alpha$-pinene tribopolymer as well as the adhesion force increased as the indentation depth was increased (Figure 5a). This result implied that $\alpha$-pinene tribopolymer is 
viscoelastic.[50] Since a constant loading rate was used, a larger indentation depth meant a longer contact time; the $\alpha$-pinene tribo-polymer had more time to flow and make greater contact with the probing tip surface. The strong adhesion to the tip surface resulted in a longer pulling of a meniscus before the tip was completely separated from the polymer surface and returned to the free standing position (zero force) during the unloading cycle.[51] This behavior is consistent with the viscoelastic property of poly- $\alpha$-pinene, which is widely used as a tackifying agent for pressure-sensitive adhesives.[23] So, the viscoelastic response observed in Figure 5b confirmed the similarity of the mechanical properties of $\alpha$-pinene tribopolymer to the chemicallysynthesized poly- $\alpha$-pinene, which is supported by the IR analysis (Figure 3a).

The pinane tribo-polymer showed more elastic behavior than the $\alpha$-pinene tribo-polymer (Figure 5b). The hysteresis between the loading and unloading curves was much smaller for the pinane tribo-polymer. This could be due to the difference in relative abundance of $p$-menthane and bornane moieties in the tribo-products of $\alpha$-pinene versus pinane. The $n$-decane tribopolymer showed much stiffer behavior than the pinane tribo-polymer (Figure 5c). Again, these differences in mechanical properties must be due to the distinct chemical structures of precursor molecules involved in tribochemical polymerization at the sliding interface.

The elastic modulus of the material probed with the AFM tip can be estimated from the stiffness of the unloading curve using the Oliver-Pharr indentation model or fitting the sample deformation with the Hertzian model.[52], [53] In these calculations, the contact area was estimated from the indentation depth and the nominal dimensions of the four-sided pyramidal AFM tip provided from the manufacturer.[53] The Poisson ratio of the tribo-polymer products is unknown; thus, for a simple comparison purpose, it was assumed to be $\sim 0.45$, which is within the typical range for rubber materials $(0.4 \sim 0.49)$. [54] Since the tribo-polymer is compliant, the 
Johnson-Kendall-Roberts (JKR) model might be more appropriate (see the Supplementary Information).[55] The elastic modulus values estimated from the force-distance curves shown in Figure 5 are listed in Table 1. Although the absolute values could not be determined because the uncertainty in the Poisson ratio and the zero-point contact position, these values chould be taken as a qualitative trend. The $\alpha$-pinene tribo-polymer still shows the lowest elastic modulus around $1 \mathrm{GPa}$, then the next is the pinane tribo-polymer, and the hardest is the n-decane tribo-polymer. The modulus value obtained for $\alpha$-pinene from the Oliver-Pharr method was comparable to the value reported for hydrogenated poly- $\beta$-pinene (2.5 GPa).[34] There is no literature value for the other two polymers since they cannot be synthesized through conventional polymerization methods.

Table 1. Elastic modulus of tribo-polymers calculated from the AFM indentation data.

\begin{tabular}{|c|c|c|c|}
\hline Polymers & From Oliver-Pharr model $^{\text {a) }}$ & From Hertzian fit ${ }^{\text {b) }}$ & From JKR fit ${ }^{\mathrm{c})}$ \\
\hline$\alpha$-pinene tribo-polymer & $1.4-1.7 \mathrm{GPa}$ & $0.5 \mathrm{GPa}$ & $1.0-1.1 \mathrm{GPa}$ \\
\hline Pinane tribo-polymer & $2.9-3.6 \mathrm{GPa}$ & $2.8 \mathrm{GPa}$ & $1.9-4.7 \mathrm{GPa}$ \\
\hline n-decane tribo-polymer & $3.5-4.2 \mathrm{GPa}$ & $7.2 \mathrm{GPa}$ & $4.8-7.3 \mathrm{GPa}$ \\
\hline
\end{tabular}

a) From the stiffness of the unloading curve of the $60 \mathrm{nN}$ and $120 \mathrm{nN}$ indentation data

b) From the loading curve of the $120 \mathrm{nN}$ indentation data

c) From unloading curve fitting of the $60 \mathrm{nN}$ and $120 \mathrm{nN}$ indentation data

The lubrication effects of these tribo-polymers in the absence of continuous supply of organic vapors were tested. The $30 \% \mathrm{p} / p_{\text {sat }} \alpha$-pinene, pinane, or $\mathrm{n}$-decane vapor was supplied for the first 1200 cycles to produce tribo-polymers and then the friction test was continued without vapor supply (Figure 6). For comparison, Figure 6 also shows the test result of n- 
pentanol VPL where the tribo-polymerization activity is negligible.[7] In the case of n-pentanol VPL, the lubrication effect was lost immediately when the n-pentanol vapor supply was ceased. In the case of tribo-polymers, when the vapor supply was stopped, the friction coefficient was slightly higher than the VPL case; but it stayed around 0.22 and stable. The monomer dissolved in the tribo-polymer would act as a plasticizer,[56-58] making the tribo-polymers more viscoelastic thus enhancing the lubrication effects of the tribo-polymer. The slight increase of the friction coefficient at the cessation of the vapor supply could be due to changes in the mechanical properties of tribo-polymer films when they lose plasticizing molecules from the bulk.

When the tribo-polymers were consumed completely, then the friction coefficient rose to 0.8 , which was the same as that in dry $\mathrm{N}_{2}$ test, indicating the lack of lubricant inside the sliding track. The lubrication effects of $\alpha$-pinene and pinane tribo-polymers were similar, although the duration was different. The $\alpha$-pinene tribo-polymers worked for 8000 cycles and then failed, while the pinane tribo-polymers functioned only for about 4000 cycles. The longer duration of the lubrication effect for the $\alpha$-pinene triboproduct could be attributed to the greater amount of the lubricating polymer films (Figure 4) and the more viscoelastic properties of the $\alpha$-pinene polymer (Figure 5). The n-decane tribo-product was not very effective at providing or maintaining lubrication effects when the n-decane vapor supply was stopped. 


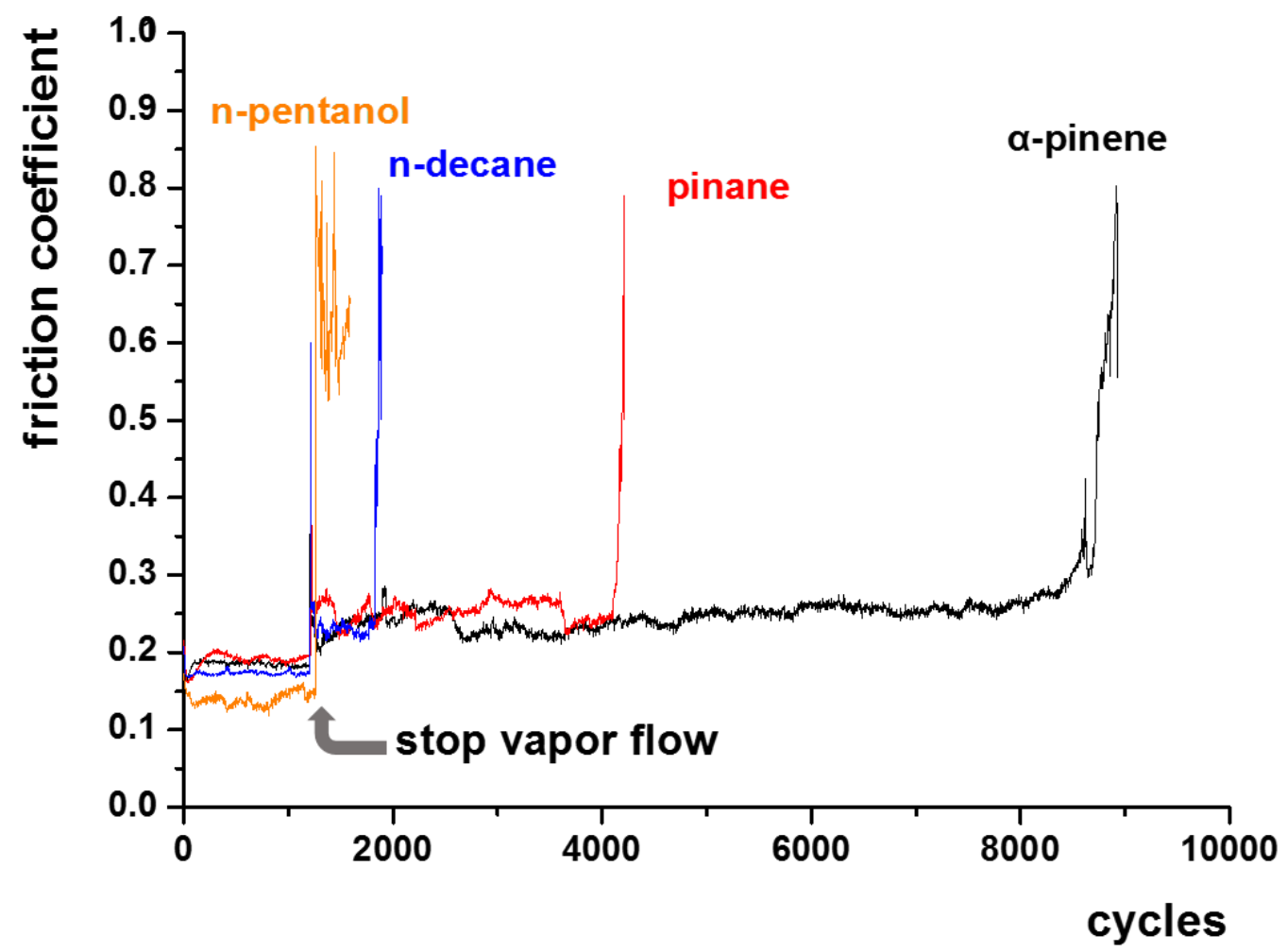

Figure 6. Lubrication effect of the tribo-polymer films in the absence of vapor supply. The tribopolymer film was synthesized by rubbing stainless steel surfaces for 1200 cycles in the presence of $30 \% \mathrm{p} / p_{\text {sat }} \alpha$-pinene (black), pinane (red), or $\mathrm{n}$-decane (blue) and then friction was measured continuously in dry $\mathrm{N}_{2}$ after stop supplying vapors. The $30 \% \mathrm{p} / p_{\text {sat }} \mathrm{n}$-pentanol (orange) is also tested for 1200 cycles and then measured in dry $\mathrm{N}_{2}$.

\section{Conclusion}

Vapor molecules physisorbed on solid surfaces can undergo mechanochemically-induced reactions during sliding in tribological processes. The vapor of bicyclic molecules, $\alpha$-pinene and pinane, was shown to produce a polymer-like film during sliding between stainless steel surfaces which worked as an effective lubricant film in the absence of the continuous supply of the 
organic vapor. The $\alpha$-pinene and pinane tribo-polymer products seemed to have molecular structure and physical properties similar to polymer produced via ring-opening reactions. The highly-strained 4-membered ring of these terpene molecules seemed to be easily activated via a mechanochemical mechanism in the tribological interface and their reaction products worked as a boundary lubrication film.

Acknowledgements. This work was supported by the National Science Foundation (Grant No. CMMI-1435766). The authors acknowledged Dr. Jordan Lerach at the Penn State Materials Characterization Laboratory for his help with ToF-SIMS analysis.

\section{References}

[1] J.A. Williams, H. Le, J. Phys. D Appl. Phys. 201 (2006) R201.

[2] R. Maboudian, W.R. Ashurst, C. Carraro, Tribol. Lett. 12 (2002) 95.

[3] S. Onclin, B.J. Ravoo, D.N. Reinhoudt, Angew. Chemie - Int. Ed. 44 (2005) 6282.

[4] D.B. Asay, M.T. Dugger, J.A. Ohlhausen, S.H. Kim, Langmuir 24 (2008) 155.

[5] A.J. Gellman, Tribol. Lett. 17 (2004) 455.

[6] S.-S. Yoo, D.-E. Kim, Int. J. Precis. Eng. Manuf. 15 (2014) 867.

[7] A.J. Barthel, S.H. Kim, Langmuir 30 (2014) 6489.

[8] K. Strawhecker, D.B. Asay, J. McKinney, S.H. Kim, Tribol. Lett. 19 (2005) 17. 
[9] C.F. McFadden, A.J. Gellman, Surf. Sci. 409 (1998) 171.

[10] D.B. Asay, M.T. Dugger, S.H. Kim, Tribol. Lett. 29 (2008) 67.

[11] C.M. Allen, E. Drauglis, Wear 14 (1969) 363.

[12] A.L. Barnette, D.B. Asay, D. Kim, B.D. Guyer, H. Lim, M.J. Janik, S.H. Kim, Langmuir 25 (2009) 13052.

[13] K.M. Wiggins, J.N. Brantley, C.W. Bielawski, ACS Macro Lett. 1 (2012) 623.

[14] N.N. Gosvami, J.A. Bares, F. Mangolini, A.R. Konicek, D.G. Yablon, Science (80-. ). 348 (2015) 102.

[15] M.K. Beyer, H. Clausen-Schaumann, Chem. Rev. 105 (2005) 2921.

[16] M.K. Beyer, J. Chem. Phys. 112 (2000) 7307.

[17] H. Spikes, W. Tysoe, Tribol. Lett. 59 (n.d.).

[18] A.L. Black, J.M. Lenhardt, S.L. Craig, J. Mater. Chem. 21 (2011) 1655.

[19] A.J. Barthel, D.R. Combs, S.H. Kim, RSC Adv. (2014) 26081.

[20] C.L. Rinsch, X.L. Chen, V. Panchalingam, R.C. Eberhart, J.H. Wang, R.B. Timmons, Langmuir 12 (1996) 2995.

[21] Z. Liu, T. Zhang, W. Zeng, H. Zhu, X. An, React. Kinet. Mech. Catal. 104 (2011) 125.

[22] T. Higashimura, Y. Deng, Makromol, Chem. 2321 (1992) 2311.

[23] A.I. Sherriff, N. College, J. Appl. Plymer Sci. 17 (1973) 3423.

[24] D.B. Asay, S.H. Kim, J. Phys. Chem. B 109 (2005) 16760. 
[25] B.J. Barner, M.J. Green, E.I. Saez, R.M. Corn, Anal. Chem. 63 (1991) 55.

[26] R.G. Greenler, J. Chem. Phys. 44 (1966) 310.

[27] R.G. Greenler, J. Chem. Phys. 50 (1969) 1963.

[28] A.J. Barthel, M.D. Gregory, S.H. Kim, Tribol. Lett. 48 (2012) 305.

[29] D.B. Asay, S.H. Kim, Langmuir 23 (2007) 12174.

[30] D.B. Asay, A.L. Barnette, S.H. Kim, J. Phys. Chem. C 113 (2009) 2128.

[31] A.J. Barthel, A. Al-Azizi, N.D. Surdyka, S.H. Kim, Langmuir 30 (2014) 2977.

[32] C.P. Green, J.E. Sader, J. Appl. Phys. 92 (2002) 6262.

[33] B. Cappella, G. Dietler, Surf. Sci. Rep. 34 (1999) 1.

[34] K. Satoh, A. Nakahara, K. Mukunoki, H. Sugiyama, H. Saito, M. Kamigaito, Polym. Chem. 5 (2014) 3222.

[35] E. Hsiao, M.J. Marino, S.H. Kim, J. Colloid Interface Sci. 352 (2010) 549.

[36] D.B. Asay, M.P. de Boer, S.H. Kim, J. Adhes. Sci. Technol. 24 (2010) 2363.

[37] A.L. Barnette, D.B. Asay, J.A. Ohlhausen, M.T. Dugger, S.H. Kim, Langmuir 26 (2010) 16299.

[38] NIST Standard Reference Database 69: NIST Chemistry WebBook.

[39] D. Kinzel, A. Stolle, B. Ondruschka, L. González, Phys. Chem. Chem. Phys. 12 (2010) 9884.

[40] K.J. Shea, T.J. Lease, J. Am. Chem. Soc. 7 (1993) 2248. 
[41] M.L. Binet, S. Commereuc, V. Verney, 36 (2000) 2133.

[42] J.F. Archard, R.A. Rowntree, Wear 128 (1988) 1.

[43] O.J. Furlong, B.P. Miller, W.T. Tysoe, Tribol. Lett. 41 (2011) 257.

[44] T.D.B. Jacobs, R.W. Carpick, Nat. Nanotechnol. 8 (2013) 108.

[45] A.J.D. Silvestre, A. Gandini, in:, Monomers, Polym. Compos. from Renew. Resour., 2008, pp. 17-38.

[46] C.A. Rezende, L.-T. Lee, F. Galembeck, Langmuir 25 (2009) 9938.

[47] B. Cappella, D. Silbernagl, Thin Solid Films 516 (2008) 1952.

[48] B. Cappella, S.K. Kaliappan, H. Sturm, Macromolecules 38 (2005) 1874.

[49] X. Cai, H. Bangert, Thin Solid Films 264 (1995) 59.

[50] A. Opdahl, S.H. Kim, T.S. Koffas, C. Marmo, G.A. Somorjai, J. Biomed. Mater. Res. A $67(2003) 350$.

[51] E. Hsiao, B.D. Veres, G.J. Tudryn, S.H. Kim, Langmuir 27 (2011) 6808.

[52] G. Pharr, W. Oliver, Mrs Bull. 17 (1992) 28.

[53] X. Xi, S.H. Kim, B. Tittmann, J. Appl. Phys. 117 (2015).

[54] P.H. Mott, C.M. Roland, Phys. Rev. B - Condens. Matter Mater. Phys. 80 (2009) 1.

[55] D.M. Ebenstein, K.J. Wahl, J. Colloid Interface Sci. 298 (2006) 652.

[56] D.F. Cadogan, C.J. Howick, in:, Kirk-Othmer Encycl. Chem. Technol., 2010, pp. 1-30. 
[57] T. Mekonnen, P. Mussone, H. Khalil, D. Bressler, J. Mater. Chem. A 1 (2013) 13379.

[58] G. Wypych, in:, Handb. Plast., 2012, pp. 165-185. 